\title{
Ultrastructural study of sciatic nerve in Ts1Cje mouse model for Down syndrome: an implication of peripheral nerve defects in hypotonia
}

Usman Bala 1,2,3, Melody Pui-Yee Leong ${ }^{2,4}$, Chai Ling Lim ${ }^{1,2}$, Hayati Kadir Shahar ${ }^{5}$, Othman Fauziah ${ }^{1}$, Mei-I Lai ${ }^{2,6}$, King-Hwa Ling ${ }^{2,3}$ and Pike-See Cheah ${ }^{1,2, *}$

${ }^{1}$ Department of Human Anatomy, Faculty of Medicine and Health Sciences, Universiti Putra Malaysia, Serdang, Selangor, Malaysia.

${ }^{2}$ Genetics and Regenerative Medicine Research Centre (GRMRC), Faculty of Medicine and Health Sciences, Universiti Putra Malaysia, Serdang, Selangor, Malaysia.

${ }^{3}$ Department of Human Anatomy, College of Medical Sciences, Gombe State University, Gombe, Nigeria.

${ }^{4}$ Department of Biomedical Sciences, Faculty of Medicine and Health Sciences, Universiti Putra Malaysia, Serdang, Selangor, Malaysia.

${ }^{5}$ Department of Community Health, Faculty of Medicine and Health Sciences, Universiti Putra Malaysia, Serdang, Selangor, Malaysia.

${ }^{6}$ Department of Pathology, Faculty of Medicine and Health Sciences, Universiti Putra Malaysia, Serdang, Selangor, Malaysia.

* Correspondence: cheahpikesee@upm.edu.my; Tel.: +603-8947 2355

Received: 16 August 2018; Accepted: 25 September 2018; Published: 16 October 2018

Edited by: Zurina Hassan (Universiti Sains Malaysia)

Reviewed by: Mohd. Farooq Shaikh (Monash University Malaysia);

Wael MY Mohamed (International Islamic University Malaysia)

https://doi.org/10.31117/neuroscirn.v1i2.17

\begin{abstract}
Trisomy 21 is chromosomal abnormality that occurs as a result of triplication of human chromosome 21 (Hsa21), a condition also known as Down syndrome (DS). Beside the intellectual disability and systems anomalies, motor dysfunction due to hypotonia has also been characterised in individuals with DS and yet, its etiology remains unclear. Ts1Cje, a mouse model for DS, has a partial trisomy (Mmu16) homology to Hsa21, is widely used for DS research. This study investigated the morphological changes and degree of myelination in sciatic nerves of the Ts1Cje mice using both light and transmission electron microscopes processed images. The result showed no morphological difference in the sciatic nerve between the Ts1Cje and WT mice. The $g$ ratio of the Ts1Cje mice was significantly $(P<0.0001)$ higher compared to that of the WT mice. Two factors are known to determine the $g$ ratio, the axonal diameter and the myelin thickness. There was no significant $(P=0.2146)$ difference in the axonal diameter between the two genotypes. Interestingly, the myelin thickness was significantly $(P<0.0001)$ thinner in nerve fibres of the Ts1Cje mice as compared to that of the WT mice. It is therefore concluded that, the hypomyelination in Ts1Cje mice may affect the conduction velocity which in turn affect their motor activity.
\end{abstract}


Keywords: Down syndrome; hypotonia; Ts1Cje mice; sciatic nerve; muscle weakness;

C2018 by Bala et al for use and distribution in accord with the Creative Commons Attribution (CC BY-NC 4.0) license (https://creativecommons.org/licenses/by-nc/4.0/), which permits unrestricted non-commercial use, distribution, and reproduction in any medium, provided the original author and source are credited.

\section{INTRODUCTION}

Trisomy 21 is chromosomal abnormality that occurs as a result of triplication of human chromosome 21 (Hsa21), a condition also known as Down syndrome (DS) [1]. The most common cause of DS is chromosomal non-disjunction [2] and it is associated with factors such as genetics, increased maternal age and low socio-economic status [2, $\underline{3}]$. Beside the intellectual disability and systems anomalies, motor dysfunction due to hypotonia has also been characterised in individuals with DS [4]. Delayed in motor function development, difficulties in motor planning and instabilities in trunk and postural control [5] together with joints stiffness [6] seen in individuals with DS have significantly affect their ability to perform some functional tasks []] thus resulting great limitations in labour productivity and socio-economic development [8]. Evidence based studies have indicated the involvement of the central nervous system (CNS) disorder [ㅁ] and the defective neuromuscular junction [10] as likely factors in manifestation of hypotonia in individuals with DS. However peripheral nerve defects cannot be simply ruled out.

Over years, different mouse models of DS have been generated and each model presents similar phenotypes seen in individuals with DS [11]. Several motor dysfunctions, such as delayed motor skill development, poor fine motor movement and impaired motor coordination have been reported in some of these mouse models [12]. The Hsa21 shares a conserved homology with 3 regions of mouse chromosomes, Mmu10, Mmu16, and Mmu 17, and each chromosome contains a segment of genes that are trisomic to a different region of Hsa21 [13].
The Ts1Cje is a mouse model for DS that carries a shorter region of Mmu16 with approximately 85 genes that are homologous to genes located on Hsa21 [14]. Over years, studies showed that the Ts1Cje exhibits features associated with DS such as brain abnormality [15]. Recent studies have indicated that, this mouse model for DS exhibits motor dysfunction with weak muscle strength, poor balance and impaired motor coordination [16]. In addition, decreased in the population of type I muscle fibre and reduced nerve conduction velocity have been observed in this mouse model [16]. Yet, the possible factors contributed to reduced nerve conduction velocity in Ts1Cje mouse model for DS has not been reported.

The sciatic nerve being the largest peripheral nerve in the body, is also the most preferred and widely chosen nerve in most neurological research and peripheral nerve-related studies [17]. This nerve was chosen in preference to the other nerves in this study mainly due to its size, location and functional diversity.

Optimal theoretical G-ratio of 0.6 is reported for a myelinated axon and it is well accepted that this ratio is optimised to achieve maximal efficiency and physiological optimization [18]. The present study aims to investigate the possible contribution of sciatic nerve defects in relation to muscle weakness in Ts1Cje mice. To understand the possible contribution of morphological changes and degree of myelination in the peripheral nerve to muscle weakness seen in individuals with DS, ultrastructural and light microscopic studies of the sciatic nerves of the Ts1Cje mice were performed. Both the morphological changes and degree of myelination in the sciatic nerve of Ts1Cje mouse model for DS were also assessed. 


\section{MATERIALS AND METHODS}

2.1 Ethical approval, animal breeding, genotyping and animal husbandry

Institutional Animal Care and Use Committee (IACUC), Faculty of Medicine and Health Sciences, Universiti Putra Malaysia (UPM) approved this study (Reference number: UPM/FPSK/PADS/BR-UUH/00494). The animals were handled in accordance with IACUC guidelines. Two groups of mice, the Ts1Cje male $(n=8)$ and wildtype (WT) (C57BL/6) male $(n=8)$ at postnatal day 60-70 were used. The mice were generated by mating Ts1Cje of C57BL/ 6 background males (obtained from Walter and Eliza Hall Institute for Medical Research, Australia) with WT C57BL/6 females. The Ts1Cje littermates were identified by polymerase chain reaction (PCR) genotyping from tail biopsy genomic DNA that amplified the Grik1 gene in WT mice, while both Grik1 and Neo genes in the Ts1Cje mouse as described [14]. The disomic littermates with the WT genotype served as the normal control. All mice were housed under controlled temperatures with a 12:12 hour light-dark cycle in the mouse room facility, Genetics and Regenerative Medicine Research Centre (GRMRC), UPM. The mice were given unlimited access to standard animal feed (Altromin 1324, Germany) and clean water ad libitum.

\subsection{Sample collection}

The mice were deeply anaesthetised with inhaled $4 \%$ $(\mathrm{v} / \mathrm{v})$ isoflurane prior to cervical dislocation to eliminate perception of pain. The sciatic nerves were harvested as described [17]. The harvested nerves (both sides) were washed with chilled $1 \mathrm{X}$ phosphate buffered saline (PBS), cut into small pieces $\left(1 \mathrm{~mm}^{2}\right)$ and fixed in $4 \%$ glutaraldehyde at room temperature for 24 hours.

\subsection{Ultra-structural assessment of the sciatic nerve}

Tissue blocks of the sciatic nerve were prepared according to the standard protocol for ultrathin sections as described [18]. A semithin coronal section (1 $\mu \mathrm{m})$ of the sciatic nerve was obtained using a diamond knife (Leica, $4 \mathrm{~mm}, 45^{\circ}$ ), and stained with toluidine blue at $\sim 70^{\circ} \mathrm{C}$ for 10 seconds. The sections were washed with distilled water and dried on hot plate (Thermolyne Nuova HP). Ultrathin coronal sections $(70 \mathrm{~nm})$ of the sciatic nerve were collected and stained with uranyl acetate and Reynolds lead citrate. Briefly, the sections were submerged in a few drops of the uranyl acetate for 20 minutes at $40^{\circ} \mathrm{C}$ followed by stream-washed in distilled water for 15 seconds. Then, sections were submerged in Reynolds lead citrate surrounded by square-arranged $\mathrm{NaOH}$ for 10 minutes, stream-washed with distilled water and allowed to dry. Ultra-structural images were obtained using transmission electron microscope (TEM) (HITACHI H-7100). Ultrastructures of the sciatic nerves such as the lamellae, mitochondria, cytoskeletal (neurofilaments and microtubules) were evaluated.

\subsection{Light microscopic assessment and $G$ ratio measurement of the sciatic nerve}

A semithin sections $(1 \mu \mathrm{m})$ were examined using a light microscope (Olympus BX51, Japan) and images were captured at $100 \mathrm{X}$ magnification through CCD digital camera (Olympus DP72, Japan). Subsequently, morphological analysis of the acquired images was carried out using Image J software (National Institutes of Health, Bethesda, MD) plug-in (g ratio calculator). The original programme was downloaded from http://cifweb.unil.ch of Cellular Imaging Facility (CIF) of the University of Lausanne. G ratio analysis was performed according to the $\mathrm{G}$ ratio Calculator 1.0 manual of CIF (Version 1.0, dated 01/03/2007). Briefly, micrograph images were pre-processed by converting them into 8-bit grayscale format and background noise was removed using a median filter for better edge detection. A user define threshold (100 to 110) was applied to binarize the images. Three slides were selected from each sample ( $n=8$ per group) and four images (from each quadrant) were obtained from each slide for measurement and analysis. All myelinated nerve fibres on each image were selected and measured. Unmyelinated and smaller (developing nerve fibres) nerve fibres were excluded from measurement. Approximately 940 to 975 nerve fibres were randomly selected from each group for morphometric measurement. All morphological 
measurement and analysis were performed based on single-blinded approach with respect to the mouse genotype. The $\mathrm{g}$ ratio was calculated based on the ratio of the inner axon (d1) to outer axon diameter (d2).

\subsection{Statistical analysis}

$\mathrm{G}$ ratio was computed and analysed using Prism7 statistical software (GraphPad Software, USA). Student's $t$-test was used to compare the numerical values obtained from the two groups/genotypes. The results were expressed as mean \pm SEM and the probability values of $P<0.05$ were considered statistically significant.

\section{RESULTS}

\subsection{Ultrastructural morphology of the sciatic nerve}

An ultrastructural analysis of the sciatic nerve fibres revealed no morphological differences between the WT (Figure 1a) and the Ts1Cje mice (Figure 1b). No axonal alteration either in the form of myelin sheath distortion or degeneration in the Ts1Cje mice. The myelin lamellae were well preserved in both genotypes
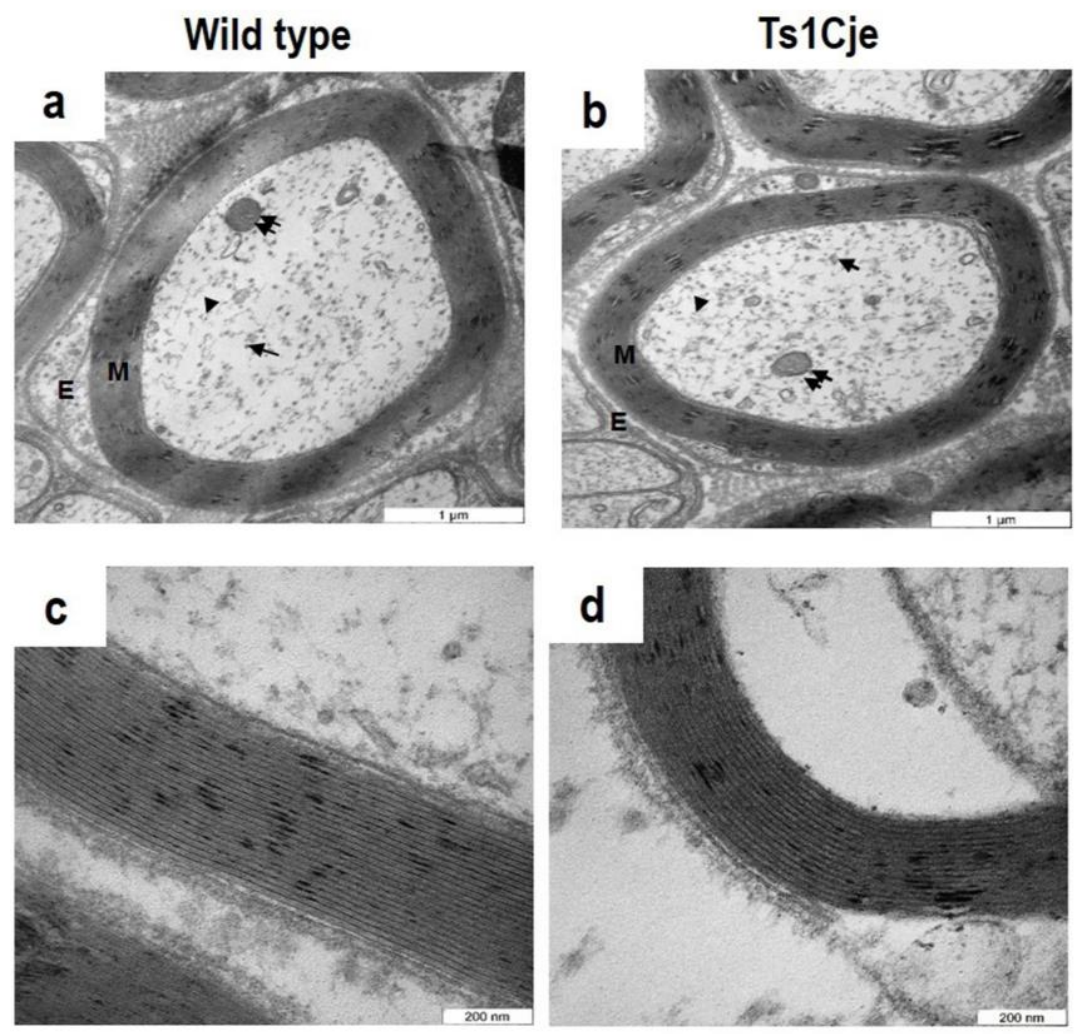

e

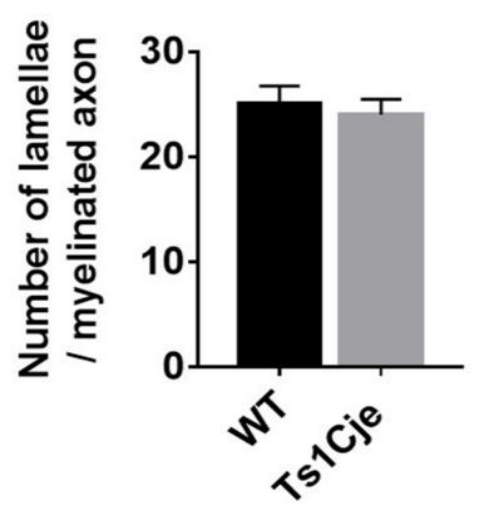

f

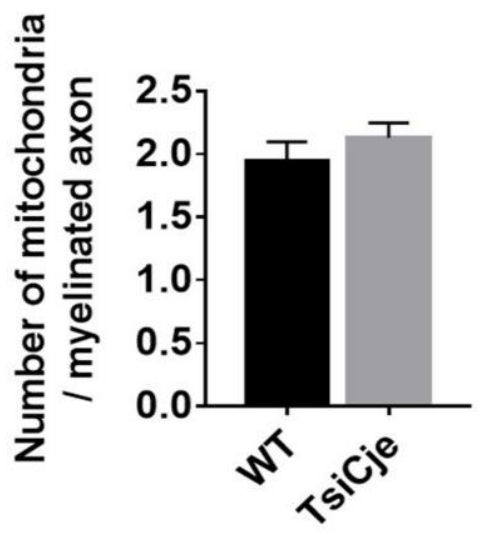

Figure 1. Evaluation of the ultrastructural morphology of the sciatic nerve. (a) Morphological observation on the coronal sections of the sciatic nerve revealed no difference in the ultrastructure of the wildtype (WT) as compared with the (b) Ts1Cje nerve fibres. High magnification of electron micrographs show the lamellae of the myelinated axons of (c) WT and (d) Ts1Cje. (e) The number of myelin sheath lamellae in Ts1Cje showed no significant difference $(P=0.46)$ from that of the WT mice. (f) No significant differences $(P=0.3381)$ in the mitochondrial population between the two genotypes.

( $M=$ =myelin; $\quad \mathrm{E}=$ =epineurium; arrow= neurofilaments; arrow head=microtubules; double arrow=mitochondria). 
[WT (Figure 1c); Ts1Cje (Figure 1d)]. The number of lamellae of individual myelinated axon was further quantified and the result showed no significant differences in the average of the lamellae between the Ts1Cje mice $(24.06 \pm 1.45, \mathrm{n}=24)$ compared to that of the WT mice (25.09 $\pm 1.70, \mathrm{n}=30, t=0.60, P=0.46)$ (Figure 1e). In addition, cytoskeletal structures such as neurofilaments and microtubules were homogeneously distributed in the nerve fibres of the Ts1Cje mice, similar to WT (data not shown). The morphology, distribution and population of the mitochondria were evaluated in the nerve fibres. There was no morphological anomaly or aggregation of mitochondria in the nerve fibres of Ts1Cje mice. Similarly, there was no significant differences in the average number of the mitochondria in the myelinated fibres between the two genotypes [(WT $=1.94 \pm 0.15$, $\mathrm{n}=54)$; (Ts1Cje=2.13 $\pm 0.12, \mathrm{n}=77, t=0.9621, P=0.3381)$ ] (Figure 1f).

\section{2 $\mathrm{G}$ ratio analysis of the sciatic nerve}

To investigate the potential role of the peripheral nerve defects in contributing to muscle weakness in DS, morphometric analysis of the sciatic nerve fibres between the two genotypes was performed using light microscopic images (Figure 2a). The g ratio was computed using g ratio calculator and the result was compared between the Ts1Cje and WT mice. According to the previous study, the optimized value of G-ratio ranged 0.55-0.68 [18]. The g ratio of the Ts1Cje mice (0.714 $\pm 0.002, n=974)$ was significantly higher compared to that of the WT mice $(0.656 \pm 0.002$, $\mathrm{n}=944, t=19.46, P<0.0001$ ) (Figure $2 \mathrm{~b}$ ). There are two major factors that affect the $\mathrm{g}$ ratio value; myelin thickness and the size of the axon. Further analysis was performed to evaluate the axonal diameter of the Ts1Cje and WT mice. The result showed no significant difference between the axonal diameter of the Ts1Cje (range $=1.08-3.31 ; 1.895 \pm 0.011 \mu \mathrm{m}, \mathrm{n}=975$ ) and WT mice (range $=0.87-3.27 ; 1.873 \pm 0.013 \mu \mathrm{m}, \mathrm{n}=943$; $t=1.335, P=0.1821$ ) (Figure 2c). Subsequent analysis indicated that the myelin thickness was significantly thinner in nerve fibres of the Ts1Cje mice (range $=0.08$ -
$0.97 ; 0.372 \pm 0.003 \mu \mathrm{m} n=971)$ as compared to that of the WT mice (range $=0.21-1.95 ; 0.487 \pm 0.004 \mu \mathrm{m}$ $\mathrm{n}=943 ; t=24.81 ; P<0.0001$ ) (Figure $2 \mathrm{~d}$ ). Scatter plots (Figures 2e-g) show the pattern of distribution of axonal diameter and myelin sheath thickness. The distribution of fibres shifted to the left in the plots of Ts1Cje and this indicates high population of the fibres with thinner myelin sheaths as compared to the WT mice.

\section{DISCUSSION}

The functional and structural index for assessing axonal myelination is the ratio of the axon diameter to the myelinated fibre diameter ( $\mathrm{g}$ ratio) and the concept of $\mathrm{g}$ ratio has been used in assessing the degree of myelination of the peripheral nerves $[\underline{19}, \underline{20}]$. Recent studies have shown that, the g ratio concept is useful in evaluating the level of myelination of the sciatic nerve during aging [21].

The axonal diameter and myelin thickness are two determinant factors for the $\mathrm{g}$ ratio. There is marked association between the myelin thickness and the axon diameter during nerve development [22]. The axonal diameter significantly influences the conduction velocity of a nerve fibres $[\underline{23}, \underline{24}]$. In myelinated fibres, changes in the size of the axon is proportional to the conduction velocity. Thus, an increase in the axonal diameter results in an increase in the conduction velocity of the nerve fibre. Nerve fibres with smaller axon diameter are slow in conduction [24]. A recent studies revealed that, Ts1Cje mice have low conduction velocity as compared with the WT [16]. In this study, the axonal diameter is relatively the same in both genotypes. Therefore, based on this finding, the possibility for the axonal diameter to influences the function of the nerve fibre in Ts1Cje mice may be excluded.

The myelin thickness is inversely proportional to the $\mathrm{g}$ ratio and plays a significant role in conduction velocity. The myelin sheath in Ts1Cje mice is thinner as compared to their aged matched WT. 


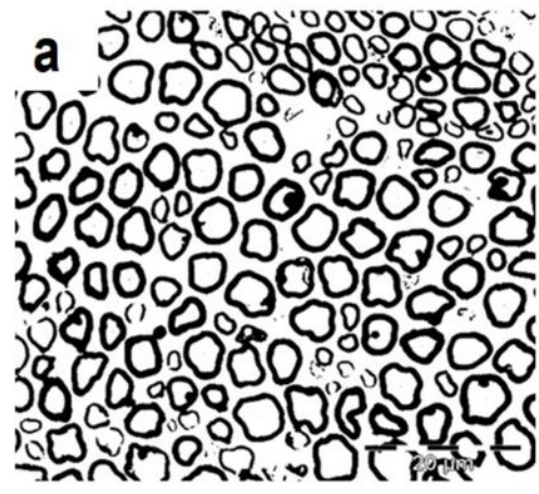

C

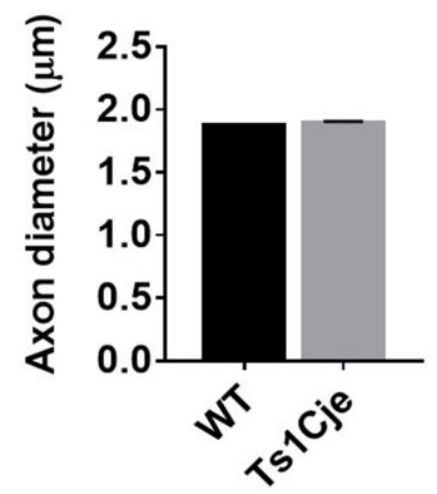

e

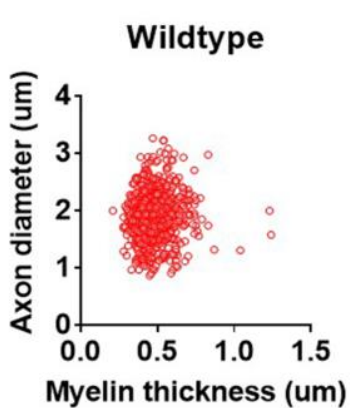

b

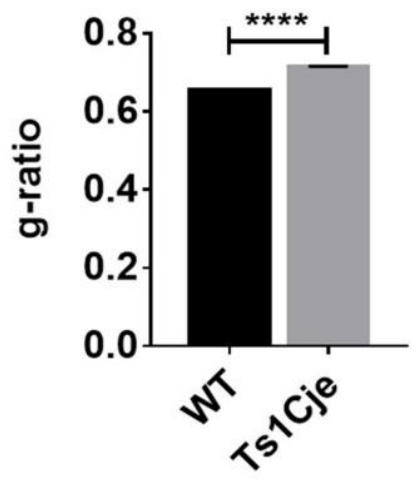

d

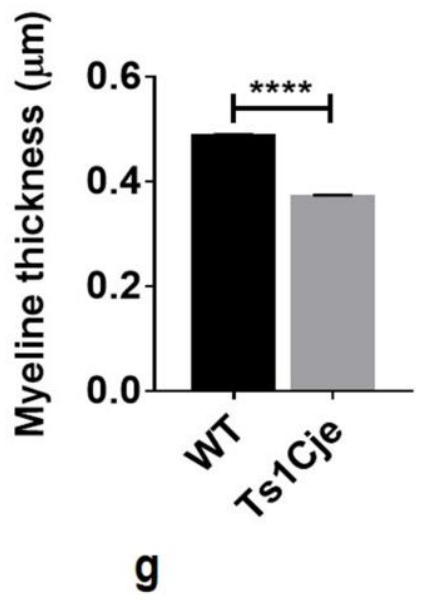

Ts1Cje

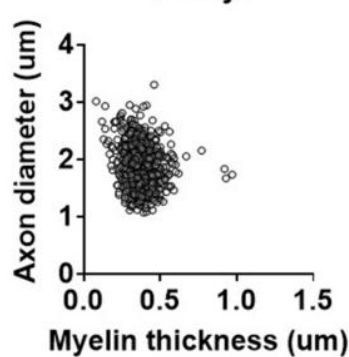

Combined Plot

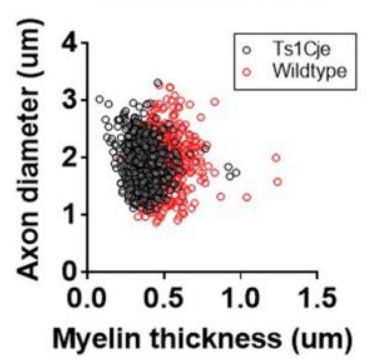

Figure 2. Evaluation of $g$ ratio and myelin thickness in the Ts1Cje mice.

(a) Semithin sections of the sciatic nerves were used for $\mathrm{g}$ ratio calculation. (b) Morphometric analysis of the nerve fibres revealed a significantly $(P<0.0001)$ higher $\mathrm{g}$ ratio in the Ts1Cje mice as compared to the WT mice. (c) The axon diameter of the Ts1Cje mice showed no significant difference ( $P=0.1821)$ from that of the WT mice. (d) Myelin sheath in WT mice was significantly thicker $(P<0.0001)$ compared to the Ts1Cje mice. (e-g) Scatter plots show the nerve fibres of both genotypes, confirming the presence of nerve fibres of Ts1Cje mice have thinner myelin sheath than that of the WT mice.
The low conduction velocity observed in the adult Ts1Cje mice [16] further support our finding of low myelin sheath in Ts1Cje mice. It is known that, myelin thickness is proportional to the conduction velocity and several studies have shown that, hypomyelination significantly affects the conduction velocity in both human diseased condition [25] and mouse model of peripheral neuropathies [26]. Thus nerve fibre with a thick myelin sheath conducts impulse faster than less myelinated or unmyelinated nerve fibre [23] The Ts1Cje mice have an intact axonal diameter but its myelin sheath was significantly thinner as compared to WT mice. It is therefore tempted to suggest that myelin is the key element responsible for the low conduction velocity in the adult Ts1Cje mice [16]. However, it may be interesting to investigate the quality of the connection between the nerve fibres and the target organ (muscle) at the neuromuscular junction. Axon extends distally to reach the appropriate target organ during nerve development. When there is an inappropriate connection between the sprouting axon with the target organ, it tends to inhibit the supply of necessary growth factors, thus resulting in defective nerve function. Similarly, to fully understand the factors that may be responsible for the low conduction in Ts1Cje mice, it is proposed that 
a couple of genes contributed in myelin development and/or maintenance such as early growth response gene 2 (Egr2) [27], myelin protein zero (MPZ) and peripheral myelin protein 22 (PMP22) [28]. Analysis of peripheral nerve genes expression profile warrant further investigation to identify the causative genes involved in defective peripheral nerve myelination of Ts1Cje.

\section{CONCLUSIONS}

The myelin sheath was significantly thinner in sciatic nerve of the Ts1Cje mice and this may affect the conduction velocity of the nerve fibres. Thus, hypomyelination in peripheral nerve of the Ts1Cje may explain the low conduction velocity and poor motor activity of the Ts1Cje mice.

Acknowledgements: This work was supported in part by funding from the MOHE Fundamental Research Grant Scheme (0401-15-1663FR), UPM Geran Putra IPT (UPM/700/2/1/GP-IPT/2013/9409500) and UPM Geran Putra IPS (UPM/700/2/1/GPIPS/2014/9448800) awarded to PSC; UPM Research University Grant Scheme (04-02-12-2102RU) and MOSTI ScienceFund (02-01-04-SF2336) awarded to KHL. MPYL and CLL were recipients of the Malaysian Ministry of Higher Education MyMaster scholarship.

Author Contributions: UB PSC and KHL, designed the experiments. UB, MPYL and CCL performed the experiments. UB, HKS, KHL and PSC analysed the data. OF, MIL co-supervised the experiment. UB, KHL and PSC drafted the manuscript.

Conflicts of Interest: The authors declare no conflict of interest.

\section{References}

1. Antonarakis SE, Lyle R, Dermitzakis ET, Reymond A, Deutsch S. Chromosome 21 and down syndrome: from genomics to pathophysiology. Nat Rev Genet. 2004;5(10):725-738. https://doi.org/10.1038/nrg1448

2. Sherman SL, Freeman SB, Allen EG, Lamb NE. Risk factors for nondisjunction of trisomy 21. Cytogenet Genome Res. 2005;111(3-4):273-280. https://doi.org/10.1159/000086900

3. Hunter JE, Allen EG, Shin M, Bean LJH, Correa A, Druschel C, et al. The association of low socioeconomic status and the risk of having a child with Down syndrome: a report from the National Down Syndrome Project. Genet Med. 2013;15(9):698-705. https://doi.org/10.1038/gim.2013.34

4. Lana-Elola E, Watson-Scales SD, Fisher EMC, Tybulewicz VLJ. Down syndrome: searching for the genetic culprits. Dis Model Mech. 2011;4(5):586-595. https://doi.org/10.1242/dmm.008078

5. Malak R, Kotwicka M, Krawczyk-Wasielewska A, Mojs E, Samborski W. Motor skills, cognitive development and balance functions of children with Down syndrome. Ann Agric Environ Med. 2013;20(4):803-806.

https://www.ncbi.nlm.nih.gov/pubmed/24364457

6. Galli M, Rigoldi C, Brunner R, Virji-Babul N, Giorgio A. Joint stiffness and gait pattern evaluation in children with Down syndrome. Gait Posture. 2008;28(3):502-506. https://doi.org/10.1016/i.gaitpost.2008.03.001

7. Kasari C, Freeman SF. Task-related social behavior in children with Down syndrome. Am J Ment Retard. 2001;106(3):253264. https://doi.org/10.1352/0895-8017(2001)1062.0.CO;2

8. Buckley S, Bird G, Sacks B. Social development for individuals with Down syndrome-An overview. Down Syndrome Education. 2002; pp. 1-44, ISBN: 9781903806210.

9. Anderson JS, Nielsen JA, Ferguson MA, Burback MC, Cox ET, Dai L, et al. Abnormal brain synchrony in Down Syndrome. Neuroimage Clin. 2013;2:703-715. https://doi.org/10.1016/j.nicl.2013.05.006

10. Chang KT, Min K-T. Upregulation of three Drosophila homologs of human chromosome 21 genes alters synaptic function: implications for Down syndrome. Proc Natl Acad Sci USA. 2009;106(40):17117-17122.

https://doi.org/10.1073/pnas.0904397106 
11. Davisson MT, Schmidt C, Akeson EC. Segmental trisomy of murine chromosome 16: a new model system for studying Down syndrome. Prog Clin Biol Res. 1990;360:263-280. https://www.ncbi.nlm.nih.gov/pubmed/2147289

12. Galante M, Jani H, Vanes L, Daniel H, Fisher EMC, Tybulewicz VLJ, et al. Impairments in motor coordination without major changes in cerebellar plasticity in the Tc1 mouse model of Down syndrome. Hum Mol Genet. 2009;18(8):14491463. https://doi.org/10.1093/hmg/ddp055

13. Galdzicki Z, Siarey R, Pearce R, Stoll J, Rapoport SI. On the cause of mental retardation in Down syndrome: extrapolation from full and segmental trisomy 16 mouse models. Brain Res Brain Res Rev. 2001;35(2):115-145. https://doi.org/10.1016/S0926-6410(00)00074-4

14. Sago H, Carlson EJ, Smith DJ, Kilbridge J, Rubin EM, Mobley WC, et al. Ts1Cje, a partial trisomy 16 mouse model for Down syndrome, exhibits learning and behavioral abnormalities. Proc Natl Acad Sci USA. 1998;95(11):6256-6261. https://doi.org/10.1073/pnas.95.11.6256

15. Olson LE, Roper RJ, Baxter LL, Carlson EJ, Epstein CJ, Reeves RH. Down syndrome mouse models Ts65Dn, Ts1Cje, and Ms1Cje/Ts65Dn exhibit variable severity of cerebellar phenotypes. Dev Dyn. 2004;230(3):581-589. https://doi.org/10.1002/dvdy.20079

16. Bala U, Leong MP-Y, Lim CL, Shahar HK, Othman F, Lai M-I, et al. Defects in nerve conduction velocity and different muscle fibre-type specificity contribute to muscle weakness in Ts1Cje Down syndrome mouse model. PLoS ONE. 2018;13(5):e0197711. https://doi.org/10.1371/journal.pone.0197711

17. Bala U, Tan K-L, Ling KH, Cheah P-S. Harvesting the maximum length of sciatic nerve from adult mice: a step-by-step approach. BMC Res Notes. 2014;7(1):714. https://doi.org/10.1186/1756-0500-7-714

18. Chomiak T. What is the optimal value of the g-ratio for myelinated fibers in the rat CNS? A theoretical approach. PLoS ONE. 2009;4(11):e7754. https://doi.org/10.1371/journal.pone.0007754

19. Maturana LG, Pierucci A, Simões GF, Vidigal M, Duek EAR, Vidal BC, et al. Enhanced peripheral nerve regeneration by the combination of a polycaprolactone tubular prosthesis and a scaffold of collagen with supramolecular organization. Brain Behav. 2013;3(4):417-430. https://doi.org/10.1002/brb3.145

20. Kato N, Matsumoto M, Kogawa M, Atkins GJ, Findlay DM, Fujikawa T, et al. Critical role of p38 MAPK for regeneration of the sciatic nerve following crush injury in vivo. J Neuroinflammation. 2013;10:1. https://doi.org/10.1186/1742-2094-10-1

21. Ugrenović S, Jovanović I, Vasović L, Kundalić B, Čukuranović R, Stefanović V. Morphometric analysis of the diameter and g-ratio of the myelinated nerve fibers of the human sciatic nerve during the aging process. Anat Sci Int. 2015;91(3):238245. https://doi.org/10.1007/s12565-015-0287-9

22. Kaplan S, Odaci E, Unal B, Sahin B, Fornaro M. Chapter 2: Development of the peripheral nerve. Int Rev Neurobiol. 2009;87:9-26. https://doi.org/10.1016/S0074-7742(09)87002-5

23. Wu LMN, Williams A, Delaney A, Sherman DL, Brophy PJ. Increasing internodal distance in myelinated nerves accelerates nerve conduction to a flat maximum. Curr Biol. 2012;22(20):1957-1961. https://doi.org/10.1016/i.cub.2012.08.025

24. Ikeda M, Oka Y. The relationship between nerve conduction velocity and fiber morphology during peripheral nerve regeneration. Brain Behav. 2012;2(4):382-390. https://doi.org/10.1002/brb3.61

25. Verhoeven K, De Jonghe P, Van de Putte T, Nelis E, Zwijsen A, Verpoorten N, et al. Slowed conduction and thin myelination of peripheral nerves associated with mutant rho Guanine-nucleotide exchange factor 10. Am J Hum Genet. 2003;73(4):926-932. https://doi.org/10.1086/378159

26. Rünker $A E$, Kobsar I, Fink $T$, Loers $G$, Tilling $T$, Putthoff $P$, et al. Pathology of a mouse mutation in peripheral myelin protein PO is characteristic of a severe and early onset form of human Charcot-Marie-Tooth type 1B disorder. The Journal of Cell Biology. 2004;165(4):565-573. https://doi.org/10.1083/icb.200402087

27. Le N, Nagarajan R, Wang JYT, Araki T, Schmidt RE, Milbrandt J. Analysis of congenital hypomyelinating Egr2Lo/Lo nerves identifies Sox2 as an inhibitor of Schwann cell differentiation and myelination. Proc Natl Acad Sci USA. 2005;102(7):25962601. https://doi.org/10.1073/pnas.0407836102 
28. Nave K-A, Sereda MW, Ehrenreich H. Mechanisms of disease: inherited demyelinating neuropathies--from basic to clinical research. Nat Clin Pract Neurol. 2007;3(8):453-464. https://doi.org/10.1038/ncpneuro0583 